\title{
Nonclinical development needs and regulatory requirements for multipurpose prevention technologies: A primer
}

Joseph W. Romano

Martha Brady

Population Council

Judy Manning

Follow this and additional works at: https://knowledgecommons.popcouncil.org/departments_sbsr-hiv

Part of the Health Services Research Commons, Immune System Diseases Commons, International Public Health Commons, Medicine and Health Commons, Pharmaceutics and Drug Design Commons, Pharmacy Administration, Policy and Regulation Commons, Virus Diseases Commons, and the Women's Health Commons How does access to this work benefit you? Let us know!

\section{Recommended Citation}

Romano, Joseph W., Martha Brady, and Judy Manning. 2012. "Nonclinical development needs and regulatory requirements for multipurpose prevention technologies: A primer." New York: Population Council. 


\title{
(2) Population Council
}

\section{Nonclinical Development Needs and Regulatory Requirements for Multipurpose Prevention Technologies: A Primer}

\author{
By Joe Romano, Martha Brady, and Judy Manning
}

As enthusiasm grows for the development of products that address sexual and reproductive health $(\mathrm{SRH})$ needs around the world, innovative development strategies are needed that are efficient, cost-effective, and consistent with likely regulatory requirements for such products. Such strategies will be even more critical as combination products are developed that seek to address multiple health indications simultaneously, and combine drugs and/ or devices that may be approved and/or experimentalthe prospect of which is truly a multidimensional regulatory puzzle.

This summary outlines key development elements necessary for various configurations of multipurpose prevention technology (MPT) products for the simultaneous prevention of Human Immunodeficiency Virus (HIV), other sexually transmitted infections (STIs), and/or pregnancy. The studies and activities described in this summary regarding drug substances (DS) and drug products (DP) are necessary to satisfy the content requirements for Section 7 of an Investigational New Drug (IND) application (Chemistry, Manufacture, Control [CMC]). For an initial IND submission, the CMC content must support the safety of the product (purity, stability, toxic impurities). Later on, DS and DP manufacture will need to satisfy International Conference on Harmonization (ICH) guidances Q8,' Q9, ${ }^{2}$ and Q I0, ${ }^{3}$ which will be part of the Quality System necessary for commercialization.

Key elements of MPT product development will be the assurance of the quality and safety of DS and DP, which can be achieved by proper preclinical (also referred to as nonclinical) evaluations of safety and toxicity, as well as by achieving necessary quality standards regarding the CMC for drug substances and drug products.

We consider key development issues up through but not including Phase I clinical studies. Clinical evaluation strategies for MPT products will be determined by several factors, including specific product configuration and properties; however, these issues are beyond the scope of this paper. Here, we focus on the nonclinical development needs and potential regulatory requirements for MPTs.

Combination pharmaceutical products have development and regulatory complexity beyond that of single-agent, singleindication products. The presence of more than one active pharmaceutical ingredient (API) increases the nonclinical, quality (CMC), and clinical development obligations for such a product. This is further compounded when a combination product targets more than one medical indication, such as MPTs designed as either drug+drug or drug+device combinations to protect against HIV, other STIs, and unintended pregnancy (see Figure I).

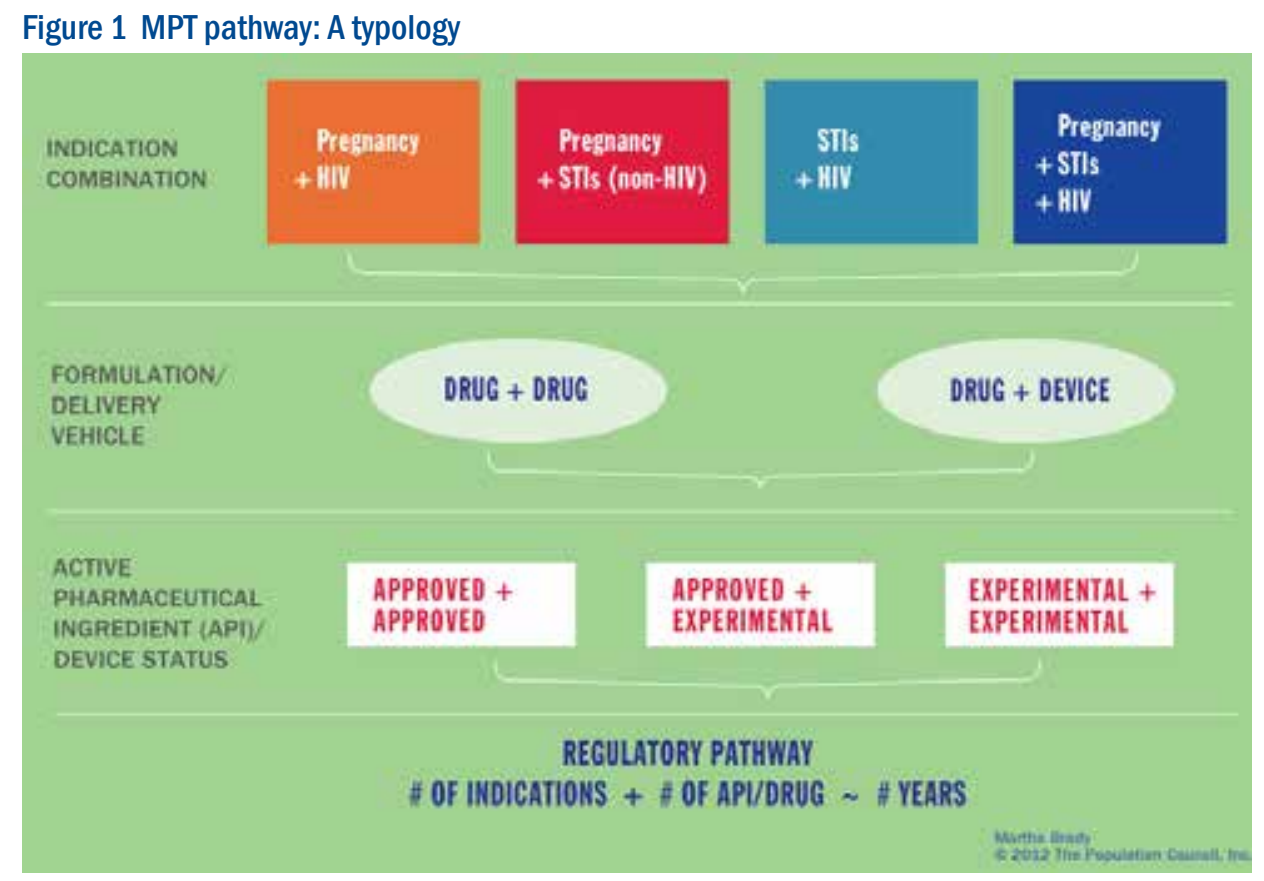




\section{Abbreviations}

$\begin{array}{ll}\text { API } & \text { Active pharmaceutical ingredient } \\ \text { ARV } & \text { Antiretroviral } \\ \text { AUC } & \text { Area under curve } \\ \text { CDER } & \text { Center for Drug Evaluation and Research } \\ \text { CDRH } & \text { Center for Devices and Radiological Health } \\ \text { C }_{\text {max }} & \text { Maximum concentration } \\ \text { CMC } & \text { Chemistry, manufacture, control } \\ \text { DOE } & \text { Design of experiment } \\ \text { DP } & \text { Drug product } \\ \text { DS } & \text { Drug substance } \\ \text { EC50 } & \text { Effective concentration, 50\% } \\ \text { EMA } & \text { European Medicines Agency } \\ \text { FDA } & \text { US Food and Drug Administration } \\ \text { GMP } & \text { Good manufacturing practice } \\ \text { HIV } & \text { Human immunodeficiency virus } \\ \text { ICH } & \text { International Conference on Harmonization } \\ \text { IND } & \text { Investigational new drug (application) } \\ \text { MoA } & \text { Mechanism of action } \\ \text { MPT } & \text { Multipurpose prevention technology } \\ \text { NDA } & \text { New drug application } \\ \text { NDE } & \text { New drug entity } \\ \text { PBMC } & \text { Peripheral blood mononuclear cells } \\ \text { PD } & \text { Pharmacodynamics } \\ \text { PEP } & \text { Post-exposure prophylaxis } \\ \text { PK } & \text { Pharmacokinetics } \\ \text { PrEP } & \text { Pre-exposure prophylaxis } \\ \text { QBD } & \text { Quality by design } \\ \text { STI } & \text { Sexually transmitted infection } \\ T_{1 / 2} & \text { Half-life; time to 50\% reduction } \\ \text { T } & \text { Time to maximum concentration } \\ & \end{array}$

\section{Acknowledgements}

The authors would like to thank David Friend of CONRAD and Jeremy Nuttall at IPM for their thorough, insightful review of this brief. We would also like to acknowledge Victor Paulus and Heeyoung Park of the Population Council for their review of an early draft.

This brief was written by Joe Romano, NWJ Group, LLC; Martha Brady, Population Council; and Judy Manning, U. S. Agency for International Development.

\section{Product Configuration Options}

Contraceptive products exist in a number of configurations and delivery strategies, including systemic formulations (oral pills, injectables, implants), topical dosage forms (gels, films, vaginal rings), intrauterine devices (with and without hormones), and physical barriers (male and female condoms, diaphragms, cervical caps). These products rely on either hormonal or nonhormonal API, and achieve effect via systemic or topical delivery. Current HIV pre-exposure prophylaxis (PrEP) products follow similar product design options for dosing and delivery, including systemic (oral, injectable), topical (gel, tablets, vaginal rings), and physical barrier (diaphragm, female condom). Although oral and topical strategies for STI prevention exist, the development of these strategies has not been as robust as HIV PrEP product development.

\section{Status of selected products}

A number of licensed contraceptive products exist, including an array of hormonal products. Although no HIV-prevention products (other than condoms) have yet to achieve regulatory licensure, a number of antiretrovirals (ARVs) approved for treatment of acute (post-exposure prophylaxis [PEP]) or chronic HIV infection are in clinical evaluation as prevention products (e.g., tenofovir, ${ }^{4}$ maraviroc $\left.^{5,6}\right)$. However, there are a number of HIVprevention candidate products in development involving API that have not been approved for treatment (e.g., MIV-I50, ${ }^{7}$ dapivirine ${ }^{8}$ ). Similarly, some early-stage product development efforts for new contraceptive agents (e.g., nonhormonal chemical entities ${ }^{9}$ ) are also pre-licensure.

MPT products can be developed from various combinations of approved and/or experimental drugs and/or devices for different single indications. The development requirements for products that do or do not involve already approved components will differ accordingly. In the case of MPTs, the variable development burdens for products with API that are in different stages of single-indication approval are potentially complicated further if the product is configured in a novel delivery device. For example, co-formulation of an approved combination oral contraceptive pill with a drug approved for treatment of HIV infection will have different development requirements and timelines than a vaginal ring designed to release an experimental nonhormonal contraceptive and an experimental ARV (see Figure 2). 
Figure 2 Optimized timelines for alternative MPT development

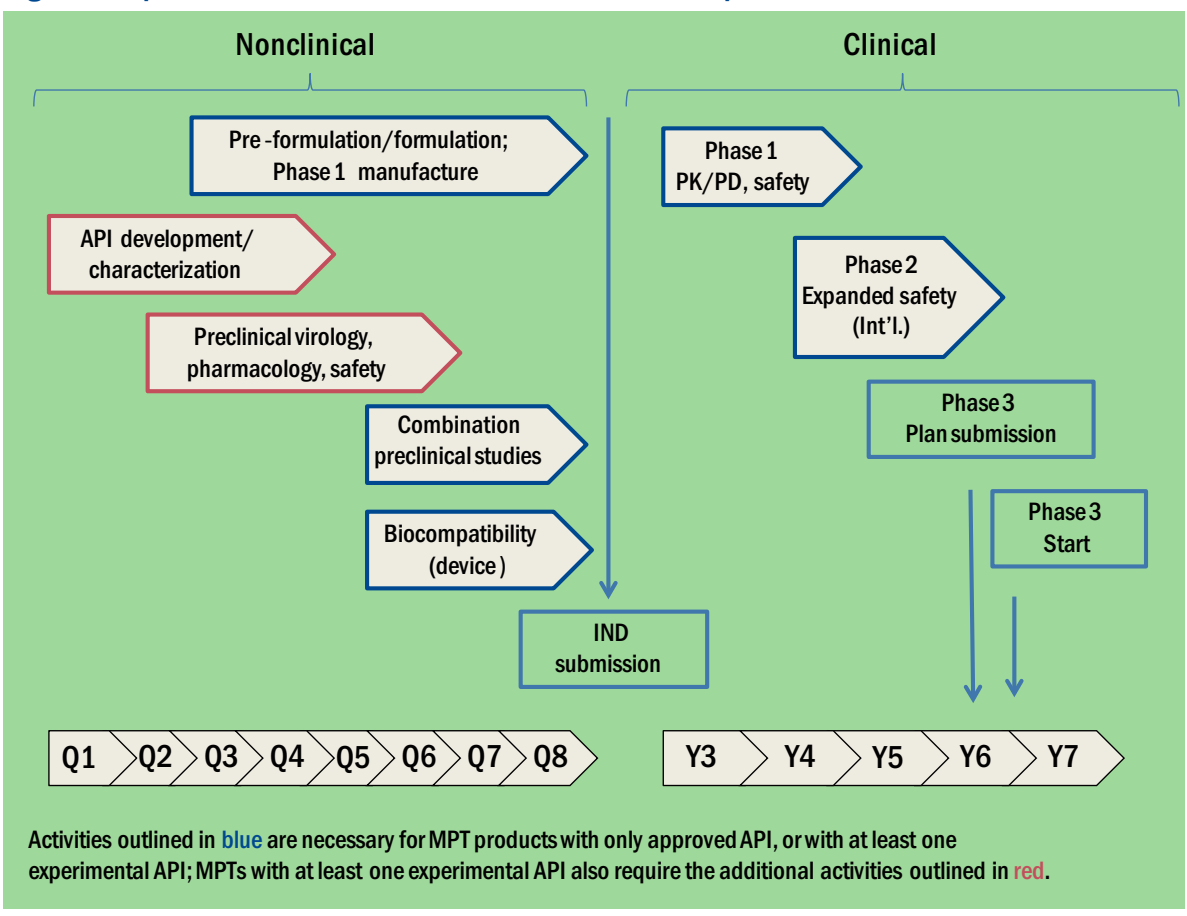

While specific development requirements will vary with each MPT product, the broad perspectives of I) experimental versus approved, 2) drug versus device, and 3) systemic versus topical delivery provide the basis for the development of general guidelines that could help to inform the broad spectrum of possible MPT product development strategies.

The following sections provide a description of development needs for possible MPT product components in terms of nonclinical safety and quality issues.

\section{MPT Case Study Number I: Co-formulation of approved drugs/ products}

\section{Nonclinical activity}

Perhaps the simplest, quickest, and least expensive MPT product development effort would involve the co-formulation of drugs and/or devices already approved for the different individual indications being targeted by the combination. (Although male and female condoms are effective HIV-prevention products, neither is currently considered a potential component of first-generation MPT products.) Further, because no drugs are yet approved for prevention of HIV, first-generation MPT products will likely involve drugs approved for treatment of HIV (or STI).

Regulators' primary concerns are the potential for: I) toxicological and 2) pharmacokinetic (PK) interactions. If there is experience of co-administration, there is no longer concern; if not, each of these should be addressed. The $\mathrm{ICH}$ guideline provides an approach for testing the toxicology interaction potential, and $\mathrm{PK}$ assessment can be included in these studies to identify PK interactions. Additionally, any product developer will want to assess potential pharmacodynamic (PD) interactions that may affect dosing.

The simplest configuration of such an MPT product would be in a dosage/delivery form similar to that already available for the individual product's specific indications (e.g., oral HIV PEP combined with an oral contraceptive). In this case, little, if any, nonclinical safety data beyond what exists for each individual product would likely be needed. The ICH M3 guideline ${ }^{10}$ suggests that where there is adequate clinical experience with co-administration of two approved late-stage products, combination toxicity studies would generally not be recommended to support clinical studies or marketing unless there is significant toxicological concern (e.g., target organ toxicity). Presumably, the individual products would have satisfied regulatory requirements for genotoxicity, acute and chronic toxicity, reprotoxicity, and so forth.

The proposed combination could possibly require a nonclinical safety assessment involving fixed plus variable doses of the two drugs in an animal system to determine if any toxicity is potentially associated with the combination. Alternatively, if an MPT product was formulated for topical use (e.g., vaginal gel or ring), additional nonclinical safety and pharmacology studies might be required, even if the product only included API from approved oral dosage forms. Aside from nonclinical safety assessments, a combination product with two (or more) approved API might also need to be evaluated from a PK perspective in an animal model. 
The purpose of this study would be to see if the presence of multiple drugs in the formulation had an effect on PK or drug distribution relative to what is observed individually with these drugs. Vaginal formulations will be required to undergo safety and PK testing, typically in a rabbit model system. This would likely involve alternative dose combinations (high, medium, low doses of API, with high, medium, low doses of $\mathrm{API}_{2}$ ). Additional vaginal exposure studies may also be needed to appropriately bridge the product formulation to data obtained from previous studies involving oral dosing (e.g., chronic toxicity, reprotoxicity).

Regarding efficacy, a justification for the product components and selected doses will be necessary. This could be achieved via a combination of in vitro, ex vivo, and possibly in vivo animal systems. Minimally, detailed in vitro infection studies with the ARV (or anti-STI) will be necessary, conducted alone and possibly in combination (depending on the product configuration) in the presence of semen and cervical-vaginal fluids. In the case of antiHIV combination products, evaluation of efficacy in the tissue explant model is also recommended." Mechanism of action (MoA) studies from the individual approved drugs should be adequate for the combination product. However, studies may also be needed to evaluate the effect of the ARV on the activity of the other component, and the effect of the other component on the activity of the ARV.

Figure 3 Potential studies to be initiated during development phases

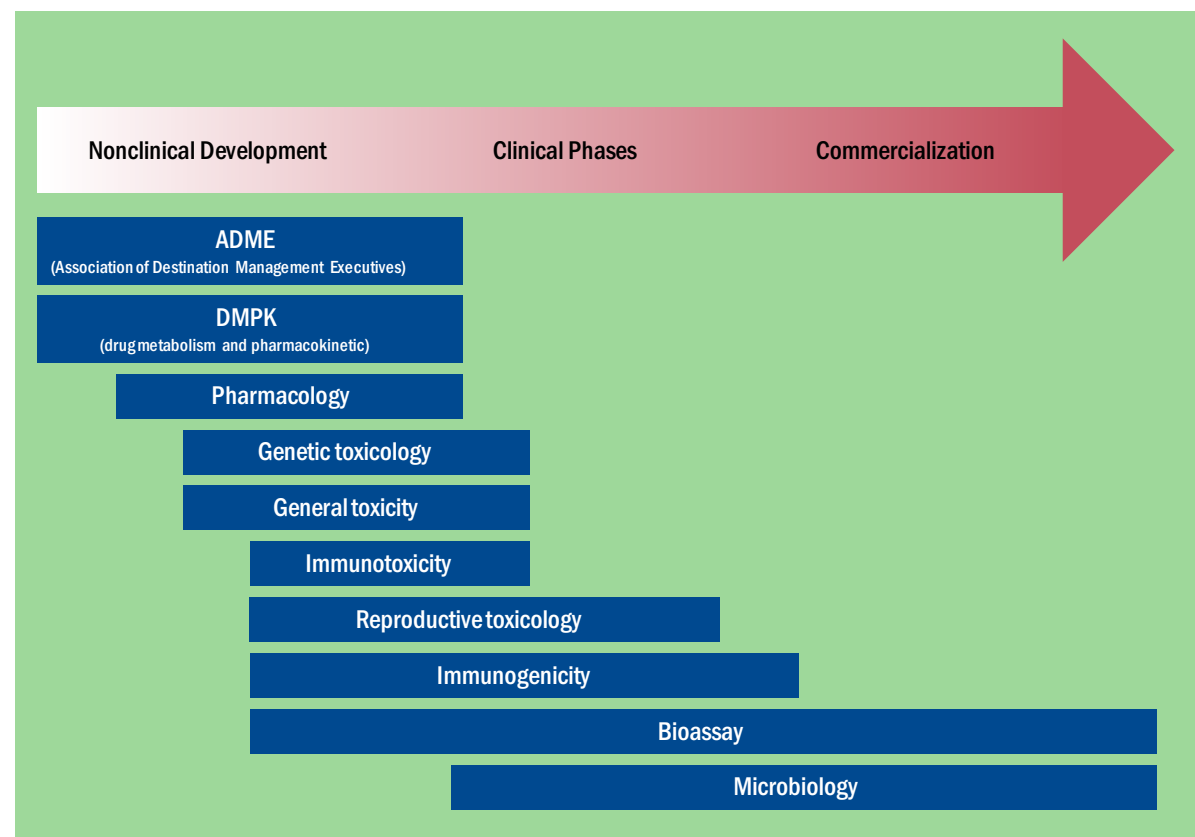

Source: Eurofins, “Integrated approach of drug development.” http://www.eurofins.com/ pharma-services/pharma-early-development.aspx. Accessed 26 September 2012.
In the case of vaginal ring delivery of combination products, $a$ biocompatibility program will be needed for a first-in-humans study. This will involve a series of extraction procedures with polar and nonpolar solvents under mild to harsh conditions. Those extracts compatible with in vitro and in vivo safety assessments will be tested in various systems to determine if elements of the device, or the devicedrug(s) combination, have a potential safety risk. The harsher condition extractions conducted with organic solvents will be necessary to look for device material degradation products, heavy metals, or other extractables creating potential safety risks for the products. Specific biocompatibility assessments need to be defined in conjunction with regulatory agencies. Similarly, although not necessary for Phase I studies with a combination product, it will be necessary to conduct formal leachables studies in the primary packaging for these products.

\section{Quality}

A major advantage of using approved drugs in an MPT combination product is that the API material has satisfied all quality requirements (i.e. CMC). Thus, the good manufacturing practice (GMP) as well as necessary analytical methods for material analysis (e.g., release and stability testing) have been adequately validated. IND applications for MPT products using approved drugs should be able to cross-reference the approved new drug application (NDA) for the API (similar to the process used to reference the approved API nonclinical and clinical data).

\section{Combination MPT products} will involve novel formulation development. The entire DP section of the IND (Section 7.2) will need to be generated. Use of approved drugs in the MPT product will not reduce the development effort or regulatory burden associated with the final product formulation. The quality requirements for an MPT product will be similar to those required for single-agent, singleindication products. However, there will likely be greater complexity on the technical development of manufacturing processes and analytical methods. 
The nature of the requirements will be a function of the product's stage of development. As the MPT product advances from Phase I through Phase 3 and on to an NDA, the quality requirements will increase and become more stringent. For early clinical work (Phase I), some of the major requirements include: formulation composition, excipient quality, manufacturing process and control, packaging, initial product specifications, qualified analytical methods for release and stability testing, stability data from multiple batches, and a certificate of analysis for clinical supplies. As the process and methods become more refined through continued development of the DP, any changes and validations will need to be documented and described. Any excipient changes (new excipients or new raw materials sources) will need to be described and qualified. Additional stability data will need to be reported as well. As the product progresses to Phase 3 , the specification limits must be narrowed and justified. Additional assessments will include: physical chemical characterization, finalization of container closure, definition of critical process parameters for design of experiment (DOE)-based quality by design (QBD) manufacturing, ${ }^{1,2,3}$ validation batch production and characterization, and so forth. In addition to the drug product material requirements, it will be necessary to develop and validate equipment cleaning procedures for the manufacturing equipment that produces the drug product.

\section{MPT Case Study Number 2:}

\section{Combination products that include one or more experimental drugs and/or devices}

Using experimental components in a new MPT product will require significantly more nonclinical and qualityrelated efforts. Typically, an experimental API, or new drug entity (NDE), is progressed through nonclinical evaluations in parallel with quality-related development. Toxicological or other issues may be identified, requiring additional follow-up studies that add cost and time to the development program. In some cases, this could result in "no go" development decisions for a product. Developing MPTs with one or more NDEs has inherently more risk and expense. This additional risk dictates the need for comprehensive nonclinical assessments and detailed quality evaluations so that major impediments to development can be identified early on, potentially saving significant effort and investment in products that are not appropriate as MPT candidates.

\section{Nonclinical evaluation of MPT products with} experimental components will require a range of nonclinical studies depending on the indication, route of administration, and physical-chemical properties of the drug andlor device. In the case of anti-HIV compounds, nonclinical virology studies will be needed. These typically include in vitro effective concentration (EC50) determinations for lab-adapted strains as well as with primary viral isolates across viral clades in peripheral blood mononuclear cells (PBMC). Other specialized assays (e.g., dendritic cell model) as well as ex vivo (human explant tissue) and in vivo (e.g., nonhuman primate) models may be part of the virology package. MoA characterization will be required as will studies looking at the development of resistance. A complete safety pharmacology package will also be required. The scope of this package will depend on whether or not the drug is administered systemically or topically (and if administered topically, how much absorption is achieved). Secondary pharmacology studies looking at other targets (e.g., enzymes, ion channels, receptors) are typically required as well. Genotoxicity will need to be assessed with at least two methods; specific systems to be evaluated might include central nervous system, cardiovascular, and/or respiratory. These assessments can be made by individual studies, or as part of a panel of more general studies including single and repeat dose toxicology studies, dose ranging studies, and long-term chronic toxicity studies (e.g., six months in dogs, nine months in rabbits). The need for and nature of these studies will be determined by data obtained through the course and stage of development, and the intended route of administration.

\section{Importance of PK Studies}

PK studies in animals are necessary for several reasons. They confirm the level of drug exposure, assuring that safety is established in the presence of the drug. They also help validate the species used in the preclinical investigations by confirming that the pharmacokinetic profiles are similar to that observed with humans. In addition, these studies enable safety margins to be established based on the higher exposures achieved in animals versus those seen in humans. In the case of MPTs, PK studies can also help define whether any interactions between APIs alter the way they are absorbed, distributed, 
metabolized, or excreted from the body that may need to be addressed in product design. The extent of PK work necessary will depend on the route of administration of the product and the extent to which the APIs are absorbed.

A PK package will be necessary, including single and repeat dose studies conducted in multiple species with plasma and tissue drug level determinations. These studies will involve standard calculations of such parameters as maximum concentration $\left(C_{\max }\right)$, time to maximum concentration $\left(T_{\max }\right)$, area under curve (AUC), and time to 50 percent reduction $\left(T_{1 / 2}\right)$. Repeat dose PK can be evaluated during toxicology studies. Distribution studies at some level will likely be required (e.g., whole body autoradiography with radiolabeled drug); however, specific metabolism and elimination study requirements will depend on the drug, route of administration, absorption, and PK profiles.

Specialized toxicology studies will be needed for MPT products, including reprotoxicity (segments I, 2, and 3), guinea pig sensitization, and other assessments depending on the drug and product configuration. This may include the biocompatibility types of studies described earlier for device-based delivery. Finally, NDE will require carcinogenicity studies in one or two species.

It is not possible to predesign a complete nonclinical package for NDE that would be necessary for NDA approval. The above summary of potential studies outlines what might be part of a typical NDE nonclinical package. Additional guidance is available ${ }^{12}$ for the nonclinical evaluation of microbicide-type products that may be useful for certain MPT products. However, the specific studies needed to support a product are to a significant degree determined through the course of the overall development of the product. Ultimately, the necessary nonclinical studies for a given product are determined through interactions with regulatory authorities.

\section{NDE quality}

The same drug product requirements discussed earlier for an MPT drug product involving approved drugs would apply to a formulated product achieved with NDE(s) as the API(s). The major difference in quality requirements for an MPT using an approved drug and an MPT using an NDE is at the level of the drug substance. Approved drugs presumably have the benefit of a full drug substance quality package; experimental drugs need to generate such a package through the course of product development.
Depending on the nature of the NDE, the drug substance quality packages could vary significantly. For example, the requirements for a small organic molecule will be quite different than those of a recombinant protein API. The requirements of either of these types of entity would differ even further from a probiotic product. Thus, it is difficult to provide a comprehensive overview of drug substance quality requirements for all possible MPT options. Minimally, a quality package for a small organic molecule API would likely require physical-chemical characterization, solubility profiles, stability assessments under different conditions (e.g., temperature, moisture, light, oxidation, etc.), route of synthesis definition, analytical methods development and validation, process scale up, and specifications with justifications. Other more specialized characterizations could include polymorph definition, sterility (if needed), and microbe testing. As in the case of drug product manufacture, experimental API production will involve definition of critical process parameters, and DOE-type process development for QBD production at commercial scale. Multiple batches of the experimental API with full stability assessments will be required, as will validation batch production and characterization. Packaging and container closure will need to be addressed for API, similar to what was described for the drug product.

MPT products can be configured with device technologies either as a means of achieving active drug delivery or as a means of directly achieving efficacy for one or more intended indications. Vaginal ring products described earlier serve as the most common example of MPT products where the device serves exclusively as the means of delivering the active pharmaceutical agents necessary for the dual indication efficacies. Typically, regulatory review of such products is achieved from the perspective of the active drugs, not the delivery mechanism. For example, primary review at the U.S. Food and Drug Administration (FDA) of a vaginal ring delivering an antiviral and hormonal contraceptive would likely occur with the Center for Drug Evaluation and Research (CDER). However, additional review of such a product would be provided by the Center for Devices and Radiological Health (CDRH). Alternatively, a device responsible directly for an efficacy outcome could be reviewed primarily by $\mathrm{CDRH}$. Agency guidance for barrier devices for contraception and STI prevention are available via the $\mathrm{CDRH} .{ }^{13}$ 


\section{Summary}

The FDA, European Medicines Agency (EMA), and ICH have produced guidance documents that provide summary information on the nonclinical and quality requirements for pharmaceutical product development. Although no specific guidance documents exist for the development of MPT products for SRH indications, a number of relevant guidance documents do exist and have been summarized elsewhere. ${ }^{14}$ Every pharmaceutical product will have its own specific requirements for development and regulatory approval, and communication with regulatory agencies is a key element of a product development effort. Understanding the requirements at different stages of product development is critical for efficient, cost effective, and successful product development. For MPT products with elevated development complexities and risks, a thorough understanding of the regulatory requirements is all the more essential.

\section{References}

I. ICH. 2009. "Harmonised tripartite guideline. Pharmaceutical development. Q8(R2).” http://www. ich.org/fileadmin/Public_Web_Site/ICH_Products/ Guidelines/Quality/Q8_RI/Step4/Q8_R2_Guideline. pdf. Accessed 5 September 2012.

2. ICH. 2005."Harmonised tripartite guideline. Quality risk management. Q9.” http://www.ich.org/fileadmin/ Public_Web_Site/ICH_Products/Guidelines/Quality/ Q9/Step4/Q9_Guideline.pdf. Accessed 5 September 2012.

3. ICH. 2008."Harmonised tripartite guideline. Pharmaceutical quality system. Q10.” http://www. ich.org/fileadmin/Public_Web_Site/ICH_Products/ Guidelines/Quality/Q10/Step4/Q10_Guideline. pdf. Accessed 5 September 2012.

4. Karim, Q.A., et al. 2010. "Effectiveness and safety of tenofovir gel, an anti-retroviral microbicide, for the prevention of HIV infection in women," Science 329: II68-1174.

5. Fletcher, P., et al. 20I0. "Anti-HIV activity of the candidate microbicide maraviroc, a CCR5 receptor agonist," abstract 21, M2010 Microbicides: Building Bridges in HIV Prevention, Pittsburgh, PA, 22-25 May 2010.

6. HIV Prevention Trials Network. "HPTN 069. Novel exploration of therapeutics for PREP." http://www. hptn.org/research_studies/hptn069.asp. Accessed 5 September 2012.

7. Kenney, J., et al. 20I I. “An antiretroviral/zinc combination gel provides 24 hours of complete protection against vaginal SHIV infection in macaques," PLoS One 6(I): el 5835.

8. Nel,A., et al. 2009."Safety and pharmacokinetics of dapivirine delivery from matrix and reservoir intravaginal rings to HIV-negative women," Journal of Acquired Immune Deficiency Syndrome 5I (4): 4I6-423.

9. Han,Y., et al. 2007. “Development of vaginal rings for sustained release of nonhormonal contraceptives and anti-HIV agents," Contraception 76(2): 132-138.

I0. ICH. 2009." "Guidance on nonclinical safety studies for the conduct of human clinical trials and marketing authorization for pharmaceuticals. M3(R2)." www. ich.org/fileadmin/Public_Web_Site/ICH_Products/ Guidelines/Multidisciplinary/M3_R2/Step4/M3_R2_ Guideline.pdf. Accessed 5 September 2012. 
I I.Sassi, A.B., et al. 20 I I. "Formulation development of retrocyclin I analog RC-IOI as an anti-HIV vaginal microbicide product," Antimicrobial Agents and Chemotherapy 55(5): 2282-2289.

12. Lard-Whiteford, S.L., et al. 2004."Recommendations for the nonclinical development of topical microbicides for the prevention of HIV transmission:An update," Journal of Acquired Immune Deficiency Syndrome 36(I): 54I-552.

13.FDA. 1990."Premarket Testing guidelines for female barrier contraceptive devices also intended to prevent sexually transmitted diseases." http:// www.fda.gov/downloads/MedicalDevices/ DeviceRegulationandGuidance/GuidanceDocuments/ UCM08 I 376.pdf?utm_campaign=Google2\&utm_ source $=$ fdaSearch\&utm_medium =website\&utm term $=$ contraceptivedevices\&utm_content=3. Accessed 5 September 2012.

I4. Brady, M., and H. Park. 20I I."What regulatory guidance exists for multipurpose prevention technologies (MPTs)? A review of key guidance documents and their applicability to MPTs," Program Brief. New York: Population Council.

\section{Other Key Resources}

FDA. "Guidance for industry: How to write a request for designation (RFD)." www.fda.gov/Regulatorylnformation/ Guidances/ucm I26053.htm. Accessed 5 September 2012.

FDA. "Guidance for industry: Codevelopment of two or more unmarketed investigational drugs for use in combination."www.fda.gov/downloads/Drugs/ GuidanceComplianceRegulatorylnformation/Guidances/ UCM236669.pdf. Accessed 5 September 2012.

FDA. "Guidance for industry and FDA staff: Early development considerations for innovative combination products. www.fda.gov/downloads/Regulatorylnformation/ Guidances/ucm I26054.pdf.Accessed 5 September 2012.

European Medicines Agency. 2009."Guideline on clinical development of fixed combination medicinal products." www.ema.europa.eu/docs/en_GB/document_library/ Scientific_guideline/2009/09/WC500003686.pdf. Accessed 5 September 2012.

This document was made possible by the generous support of the American people through the United States Agency for International Development (USAID) under the terms of Cooperative Agreement No. AID-OAA-A-10-00072. The contents are the responsibility of the authors and do not necessarily reflect the views of USAID or the United States Government.

\section{Population Council}

The Population Council confronts critical health and development issues-from stopping the spread of HIV to improving reproductive health and ensuring that young people lead full and productive lives. Through biomedical, social science, and public health research in 50 countries, we work with our partners to deliver solutions that lead to more effective policies, programs, and technologies that improve lives around the world. Established in 1952 and headquartered in New York, the Council is a nongovernmental, nonprofit organization governed by an international board of trustees. www.popcouncil.org

Suggested citation: Romano, Joe, Martha Brady, and Judy Manning. 2012."Nonclinical development needs and regulatory requirements for multipurpose prevention technologies: a primer." New York: Population Council. 